\title{
Pediatric pulmonary rehabilitation program at a Chilean public hospital
}

Gerardo Torres, Kinesiologista,b, Iván Rodríguez-Núnez, M.D. ${ }^{b}$, Daniel Zenteno, M.D. ${ }^{a, c}$,
Ximena Navarro, Kinesiologist ${ }^{a}$, Kimberly Medina, Kinesiologist ${ }^{b}$ and Jaime Tapia, M.D.

\section{ABSTRACT}

Introduction. Pediatric pulmonary rehabilitation is relevant in the management of chronic respiratory diseases. In Chile, it is provided only at certain hospitals.

Objective. To describe the outcomes of a pediatric pulmonary rehabilitation program after 3 months of rehabilitation.

Methodology. Retrospective study of patients with chronic lung disease, neuromuscular disease, and other chronic conditions admitted to the program between June 2011 and June 2017. Assessments included general physical and respiratory muscle examination, and spirometry. General physical training and respiratory muscle training were planned according to facilitybased, mixed and home protocols.

Results. A total of 156 patients ( $11.45 \pm 3.55$ years old) were included, 68 had chronic lung disease $(11.56 \pm 4.6$ years old, $43.5 \%) ; 45$, neuromuscular disease $(11.4 \pm 3.7$ years old, $28.8 \%)$; and 43 , various chronic conditions (11.31 \pm 3.9 years old). Out of all patients, $102(65.4 \%)$ followed training protocols; targeted $(n=27)$, mixed $(n=23)$, and home $(\mathrm{n}=50)$; compliance was $69 \%, 87.5 \%$, and $70 \%$, respectively. Peak inspiratory pressure increased by $19.3 \%(p=0.001)$; peak expiratory pressure, $14.5 \%(p=0.001)$; forced vital capacity, $12.9 \%(p=0.001)$; forced expiratory volume in 1 second, $11.6 \%(p=0.004)$; and the six-minute walk test, 17.6 meters $(p=0.036)$ after 3 months of rehabilitation.

Conclusions. The intervention protocol improved cardiorespiratory functional capacity. Compliance was over $65 \%$.

Keywords: musclestrength, neuromuscular diseases, lung diseases, rehabilitation, pediatrics.

http: / / dx.doi.org/10.5546/ aap.2019.eng.e576

To cite: Torres G, Rodríguez-Núñez I, Zenteno D, Navarro $X$, et al. Pediatric pulmonary rehabilitation program at a Chilean publichospital. Arch Argent Pediatr 2019;117(6):e576-e583.
E-mail address:

Gerardo Torres, B.S.:

klgo.gerardo.torres@

gmail.com

Funding:

None.

Conflict of interest:

None.

Received: 7-17-2018

Accepted: 5-8-2019

\section{GLOSSARY}

6MWT: six-minute walk test.

CLD: chronic lung disease.

$\mathrm{FEF}_{25-75}$ : mean forced expiratory flow. $\mathrm{FEV}_{1}$ : forced expiratory volume in

1 second.

FVC: forced vital capacity.

GPT: general physical training.

HGGB: Hospital Dr. Guillermo Grant

Benavente.

HR: heart rate.

NMD: neuromuscular disease.

MCF: maximal cough flow.

MEF: maximal expiratory flow.

MEP: maximal expiratory pressure.

MIP: maximal inspiratory pressure.

PR: pulmonary rehabilitation.

RMT: respiratory muscle training.

TDW: total distance walked.

\section{INTRODUCTION}

Pulmonary rehabilitation (PR) is an interdisciplinary intervention aimed at patients with chronic conditions. ${ }^{1}$ Its objective is to reduce symptoms, enhance functional capacity, increase social engagement, and reduce health costs. ${ }^{2,3}$

The development of a PR program involves coordinating patient assessment, treatment, and followup strategies taking into account the extent of functional impairment secondary to lung damage and/ or neuromuscular disease (NMD), as well as individual psychosocial aspects. ${ }^{3,4}$

Current evidence-based consensuses and guidelines have helped to establish intervention protocols associated with the assessment and rehabilitation of patients receiving PR and minimum standards for the development of this type of interventions in the clinical setting. ${ }^{1-3}$ In Chile, only few public 
facilities have developed an administrative basis to implement PR in pediatric patients with chronic conditions. One of those is the Pediatric Pulmonary Rehabilitation Program of Hospital Dr. Guillermo Grant Benavente (HGGB) in Concepción, Chile. It includes specific assessment, rehabilitation, and follow-up strategies based on each patient's biodemographic characteristics and pathophysiological aspects.

The program's clinical performance has not been reported to date, so the objective of this study was to describe the outcomes after 3 months of rehabilitation.

\section{MATERIAL AND METHOD}

Study design: retrospective case series.

Study sample: all patients with chronic lung disease (CLD), NMD, and other chronic conditions, admitted to the Pediatric Pulmonary Rehabilitation Program of HGGB between June 2011 and June 2017. Patients with cognitive deficit, younger than 5 years old or experiencing an acute respiratory condition in the 4 weeks prior to admission to the program were excluded.

\section{Pediatric pulmonary rehabilitation protocol}

The pediatric PR protocol of HGGB consists in interventions for the assessment and treatment of patients with chronic conditions (Figure 1).

\section{Assessment protocol}

Age, sex, height, and weight, measured using a tape measure and an analog scale, were recorded during admission to the program. Walking patients performed the six-minute walk test (6MWT) and had an assessment of maximal inspiratory pressure

FIGURE 1. Flowchart of referral and care at the pediatric pulmonary rehabilitation clinic of Hospital Dr. Guillermo Grant Benavente in Concepción, Chile

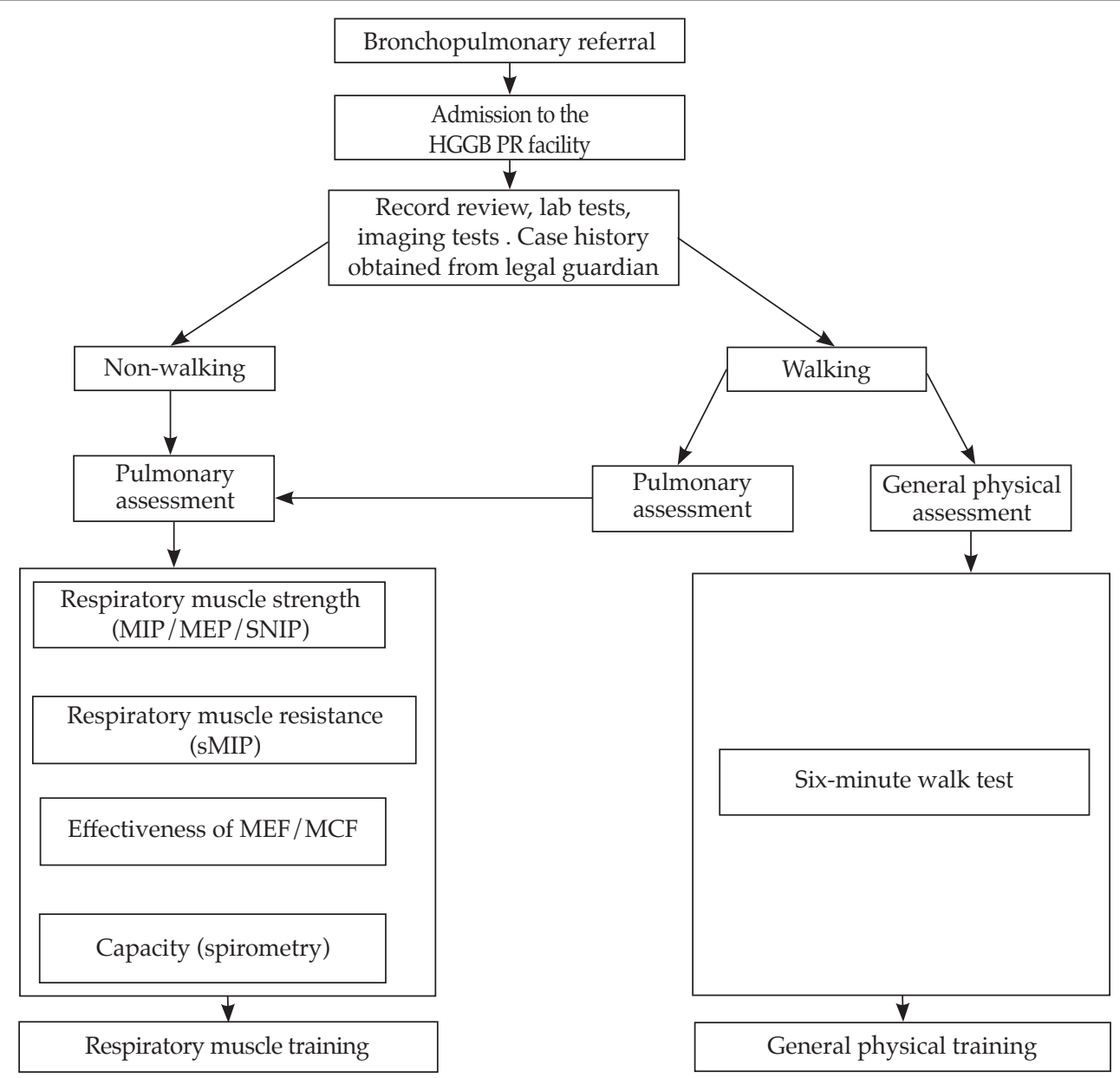

PR: pulmonary rehabilitation; HGGB: Hospital Dr. Guillermo Grant Benavente; MIP: maximal inspiratory pressure; MEP: maximal expiratory pressure; SNIP: sniff nasal inspiratory pressure; sMIP: sustained maximal inspiratory pressure; MEF: maximal expiratory flow; MCF: maximal cough flow. 
(MIP), maximal expiratory pressure (MEP), a flowmetry, and a spirometry. Patients who could not walk only had an assessment of MIP, MEP, a flowmetry and a spirometry. Each patient's parent or caregiver signed an informed consent, and children older than 12 years signed an informed assent for admission to the program and data use for scientific purposes.

6MWT: it was done according to the American Thoracic Society guidelines. ${ }^{5}$ The following information was recorded in a database: total distance walked (TDW), heart rate (HR) at rest and at the end of the 6MWT (Choicemmed ${ }^{\circledR}$ pulse oximeter), and perceived exertion based on the EPInfant scale, before and after the test. ${ }^{6}$ The TDW was recorded in absolute values $(\mathrm{m})$ and percentage as per the Chilean predictive values published by Gatica et al. ${ }^{7}$

Respiratory muscle strength assessment: MIP and MEP were measured according to the European Respiratory Society and the American Thoracic Society protocols. ${ }^{8}$ Ten attempts were made and the best value out of 3 acceptable and reproducible attempts was considered, with a variability $<5 \%$ and a 30 -second resting period between each attempt. Results were described as absolute values $\left(\mathrm{cmH}_{2} \mathrm{O}\right)$ and percentage, as per the Chilean predictive values published by Contreras et al. ${ }^{9}$ Measurements were made using a MicroRPM digital vacuum/ pressure gauge (Carefusion $^{\circledR}$ ).

Lung function assessment: it was assessed with a spirometry, according to the European Respiratory Society and the American Thoracic Society protocols. ${ }^{10}$ The following parameters were recorded: forced expiratory volume in 1 second $\left(\mathrm{FEV}_{1}\right)$, forced vital capacity (FVC), $\mathrm{FEV}_{1} / \mathrm{FVC}$ ratio, and mean forced expiratory flow $\left(\mathrm{FEF}_{25-75}\right)$. Results were described as absolute values and percentage of the predictive value as per Knudson et al., using a portable spirometer (Pony FX, Cosmed ${ }^{\circledR}$ ).

Flowmetry assessment: maximal expiratory flow (MEF) and maximal cough flow (MCF) were measured using a flow meter (Mini-Wright ${ }^{\circledR}$ ). MEF was measured with the subject standing and wearing a nose clip, and the indication was given to blow with the greatest force from total lung capacity. The test was performed a maximum of 8 times; the highest reproducible value in 3 attempts with a difference no greater than $10 \%$ between each value was recorded. ${ }^{11} \mathrm{MCF}$ was measured with the subject seated and asked to inhale deeply and then to perform a maximum coughing effort through the flow meter; results were described as liter/minute.

\section{Intervention protocol}

The patients with assessment results that were below the predicted value were admitted to the training protocol.

General physical training (GPT) was performed at $60 \%$ of HR reserve, in a continuous manner, 2-3 times per week, for 30 minutes. A treadmill (Life Fitness ${ }^{\circledR}$ T9i) was used for the training program developed at the HGGB; at home, it was performed after providing counseling to parents about exercise intensity and modality in the home setting.

In addition, respiratory muscle training (RMT) was done using a threshold valve (Threshold IMT, Philips Respironics ${ }^{\circledR}$ ). Thus, inspiratory RMT was done using a load equal to $30 \%$ of the MIP, in 3 series of 3 minutes of exercise and 1 minute of rest. Expiratory RMT was done in 3 series of $15-20$ repetitions at $50 \%$ of the MEP, with 1 minute of rest, 5 times per week both at the rehabilitation facility and at home, as per the different consensuses. ${ }^{1,12,13}$

Follow-up and compliance: for the follow-up during PR, 3 customized strategies were established based on geographic and socioeconomic variables: (a) Targeted protocol (assessments, RMT and GPT 2-3 times per week at the hospital's PR facility); (b) mixed protocol (RMT at home and 2-3 controls per month, with GPT both at the hospital and at home); and (c) unsupervised home protocol (home RMT, 1-2 controls every 6 months at the hospital, and GPT entirely at home). The patients who had access to the facility and adequate financial means performed the targeted or mixed protocol; whereas those who did not, performed the home protocol.

The protocol outcomes were established based on the outcome measures obtained 12 weeks after admission to the PR program.

RMT compliance was established based on the information provided by the patient's parents or legal guardian. Compliance was considered adequate if training was performed 5 times a week. On the other side, GPT compliance was defined only in the group that performed the targeted protocol, and adequate compliance was established if the patient attended the scheduled sessions continuously for at least 8 weeks.

Statistical analysis: descriptive statistics were established using average and standard 
deviation for quantitative outcome measures and percentage for qualitative outcome measures.

The general characteristics of subjects with CLD, NMD, and other chronic conditions were analyzed; a one-way analysis of variance (ANOVA) was done for biodemographic outcome measures and a $\chi^{2}$ test, for the sex outcome measure.

CLD and NMD patient groups were compared; Student's $t$ test was done for paired samples and the Wilcoxon test was used to compare pre- and post-intervention protocol assessments. In addition, the Mann-Whitney U test and Student's t test were done for independent samples to compare NMD and CLD.

Training outcomes were measured by analyzing the differences between pre- and post-intervention values of assessed outcome measures. The analysis was done with the SPSS v.23.0 statistical software package; a value of $p<0.05$ was considered significant.
Ethical considerations: the study was approved by the Scientific Ethics Committee of the HGGB.

\section{RESULTS}

In the study period, a total of 156 patients were included; $102(65.4 \%)$ showed lower than predicted values in the assessments, so they performed some type of training.

No differences were observed among the CLD, NMD, and other chronic conditions groups in terms of age, sex, and anthropometric outcome measures (Table 1). Patients in the NMD group mainly had Duchenne muscular dystrophy $(n=13)$, myelomeningocele $(n=10)$, and spinal muscular atrophy (SMA) type 2 $(n=5)$; whereas those in the CLD group had obliterative bronchiolitis $(\mathrm{n}=27)$, cystic fibrosis $(\mathrm{n}=19)$, and asthma $(\mathrm{n}=15)$, among others. Other chronic conditions included sleep apnea syndrome, obesity, severe scoliosis, and heart

TABLE 1. General characteristics of studied groups, described as mean \pm standard deviation

\begin{tabular}{lcccc}
\hline Outcome measures & $\begin{array}{c}\text { Total } \\
(\mathbf{n = 1 5 6 )}\end{array}$ & $\begin{array}{c}\text { NMD } \\
(\mathbf{n}=\mathbf{4 5})\end{array}$ & $\begin{array}{c}\text { CLD } \\
(\mathbf{n}=\mathbf{6 8})\end{array}$ & $\begin{array}{c}\text { Other chronic conditions } \\
(\mathbf{n}=\mathbf{4 3})\end{array}$ \\
\hline Age (years old) & $11.5 \pm 3.6$ & $11.6 \pm 4.6$ & $11.4 \pm 3.7$ & $11.3 \pm 3.9$ \\
Sex (M/F) & $91 / 65$ & $29 / 16$ & $41 / 27$ & $21 / 22$ \\
Weight (kg) & $40.1 \pm 17.6$ & $38.3 \pm 17.7$ & $39.8 \pm 15.3$ & 0.371 \\
Height (cm) & $138.1 \pm 21.2$ & $134.2 \pm 19.0$ & $140.5 \pm 21.7$ & $0.302^{\mathrm{b}}$ \\
BMI & $20.3 \pm 5.3$ & $20.6 \pm 5.9$ & $19.6 \pm 4.1$ & 0.577 \\
\hline
\end{tabular}

BMI: body mass index; M: male; F: female; NMD: neuromuscular disease; CLD: chronic lung disease.

${ }^{\mathrm{a}}$ One-way ANOVA; ${ }^{\mathrm{b}} \chi^{2}$ test.

TABLE 2. Baseline assessment of respiratory muscle function, flowmetry, spirometry, and six-minute walk test for the total group and for neuromuscular disease and chronic lung disease sub-groups, described as mean \pm standard deviation

\begin{tabular}{|c|c|c|c|c|}
\hline Outcome measures & $\begin{array}{c}\text { Total } \\
(\mathrm{n}=156)\end{array}$ & $\begin{array}{c}\text { NMD } \\
(n=45)\end{array}$ & $\begin{array}{c}\text { CLD } \\
(n=68)\end{array}$ & $p$ value $^{b}$ \\
\hline CVF (L) & $1,91 \pm 0,95$ & $1,68 \pm 0,69$ & $2,20 \pm 1,06$ & 0,083 \\
\hline FVC (L) & $1.91 \pm 0.95$ & $1.68 \pm 0.69$ & $2.20 \pm 1.06$ & 0.083 \\
\hline $\mathrm{FVC}(\%)$ & $87.7 \pm 27.7$ & $84.9 \pm 33.5$ & $92.7 \pm 21.6$ & 0.382 \\
\hline $\mathrm{FEV}_{1}(\mathrm{~L})$ & $1.51 \pm 0.70$ & $1.51 \pm 0.54$ & $1.63 \pm 0.79$ & $0.512^{\mathrm{a}}$ \\
\hline $\mathrm{FEV}_{1}(\%)$ & $77.9 \pm 25.8$ & $83.4 \pm 30.8$ & $76.1 \pm 21.7$ & $0.052^{\mathrm{a}}$ \\
\hline $\mathrm{FEV}_{1} / \mathrm{FVC}$ & $77.4 \pm 22.2$ & $89.6 \pm 8.3$ & $75.0 \pm 13.0$ & $0.001^{* a}$ \\
\hline $\mathrm{FEF}_{25-75}(\mathrm{~L} / \mathrm{min})$ & $1.62 \pm 0.79$ & $1.97 \pm 0.70$ & $1.49 \pm 0.87$ & $0.004^{*_{a}}$ \\
\hline $\mathrm{FEF}_{25-75}(\%)$ & $66.7 \pm 33.5$ & $93.6 \pm 29.2$ & $53.7 \pm 31.9$ & $0.001^{*_{a}}$ \\
\hline $\mathrm{MIP}\left(\mathrm{cmH}_{2} \mathrm{O}\right)$ & $59.6 \pm 20.4$ & $55.3 \pm 19.7$ & $66.2 \pm 19.0$ & $0.010^{*}$ \\
\hline $\operatorname{MIP}(\%)$ & $57.4 \pm 20.5$ & $51.7 \pm 17.5$ & $64.3 \pm 19.8$ & $0.006^{*}$ \\
\hline $\operatorname{MEP}\left(\mathrm{cmH}_{2} \mathrm{O}\right)$ & $58.0 \pm 24.5$ & $42.6 \pm 18.4$ & $70.5 \pm 21.6$ & $0.001^{*}$ \\
\hline $\operatorname{MEP}(\%)$ & $43.7 \pm 18.0$ & $32.2 \pm 14.4$ & $52.6 \pm 17.0$ & $0.001^{* a}$ \\
\hline MEF (L/min) & $203.2 \pm 102.5$ & $150.36 \pm 51.3$ & $230.3 \pm 107.2$ & 0.331 \\
\hline $\operatorname{MEFc}(\mathrm{L} / \mathrm{min})$ & $198 \pm 128.2$ & $165.0 \pm 56.4$ & $217.7 \pm 84.2$ & 0.318 \\
\hline 6MWT (m) & $513.9 \pm 132.2$ & $359.8 \pm 138.2$ & $564.6 \pm 89.7$ & $0.001^{*}$ \\
\hline 6MWT $(\%)$ & $81.5 \pm 16.7$ & $62.3 \pm 18.9$ & $67.0 \pm 20.7$ & 0.001 \\
\hline
\end{tabular}

*MIP: maximal inspiratory pressure; MEP: maximal expiratory pressure; MEF: maximal expiratory flow;

MEFc: maximal expiratory flow coughed; 6MWT: six-minute walk test; NMD: neuromuscular disease; CLD: chronic lung disease.

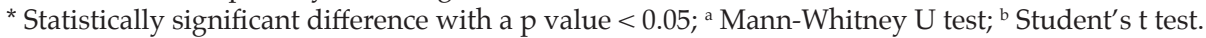


disease. No patient in the sample had respiratory insufficiency with oxygen requirement.

Out of 102 patients who received training, 38 performed only RMT; 27, only GPT; and 37, RMT/GPT. Among those who performed RMT, $34(45 \%)$ had NMD and $23(31 \%)$, CLD $(p=0.001)$; whereas among those who did GPT, 12 $(19 \%)$ had NMD and $37(58 \%)$, CLD $(p=0.001)$.

In relation to the training modality, 27 patients $(19.4 \%)$ followed the targeted protocol; 23 $(16.5 \%)$, mixed training, and $50(40.0 \%)$, home training, with a compliance of $69.0 \%, 87.5 \%$, and $70.0 \%$, respectively.

In relation to respiratory muscle strength, both groups had MIP and MEP values below the theoretical normal value (Table 2). An obstructive ventilatory pattern was observed in children with CLD. On their side, NMD patients had a normal ventilatory pattern.

\section{Intervention protocol outcomes on pulmonary function parameters and general physical capacity}

Figure 2 shows the post-PR changes in respiratory muscle strength, flowmetry, and walk test in the total group with training $(\mathrm{n}=102)$.

In relation to respiratory muscle strength, MIP was observed to increase by $11.5 \mathrm{cmH}_{2} \mathrm{O}(19.3 \%$; $p=0.001)$ and MEP, $8.41 \mathrm{cmH}_{2} \mathrm{O}(14.5 \% ; p=0.001)$. In the spirometry, FVC increased by 0.246 liters $(12.9 \% ; p=0.001)$, while $\mathrm{FEV}_{1}, 0.177$ liters $(11.6 \%$; $p=0.004$ ) (Table 3 ). In addition, the TDW in the 6MWT increased by 17.6 meters $(3.4 \% ; p=0.036)$.

The analysis of the NMD and CLD groups showed that patients with NMD had an improvement of $7.66 \mathrm{cmH}_{2} \mathrm{O}$ in their MEP $(17.9 \% ; p=0.014)$ and of $22.2 \mathrm{~L} / \mathrm{min}$ in their PCF $(18.8 \% ; p=0.021)$. Patients with CLD had an improvement of 0.396 liters in their FVC (17.9 \%; $p=0.001)$, of 0.325 liters in their $\mathrm{FEV}_{1}(19.9 \%$; $p=0.001)$, and of $11.7 \mathrm{cmH}_{2} \mathrm{O}$ in their MIP $(17.6 \%$; $p=0.006)$ (Table 4).

\section{DISCUSSION}

The objective of this study was to describe the characteristics of a PR program for children and adolescents with chronic conditions at a Chilean public hospital. Patients were observed to experience significant improvements in respiratory muscle strength, lung volumes and capacity, and a longer TDW in the 6MWT. These outcomes were consistent with those that had revealed the benefits of RMT and GPT on the function of respiratory muscles, spirometry
FIGURE 2. Diagrams for respiratory muscle strength (A), flowmetry (B), and distance walked in the six-minute walk test (C) at baseline and 3 months after admission for the total group with training $(n=102)$

A

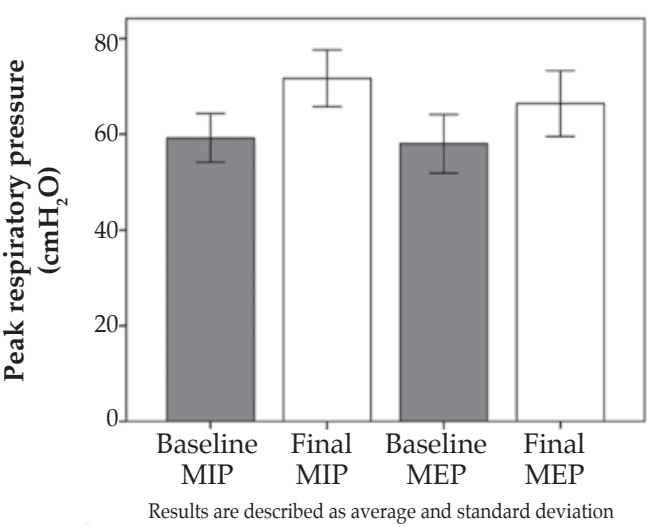

B

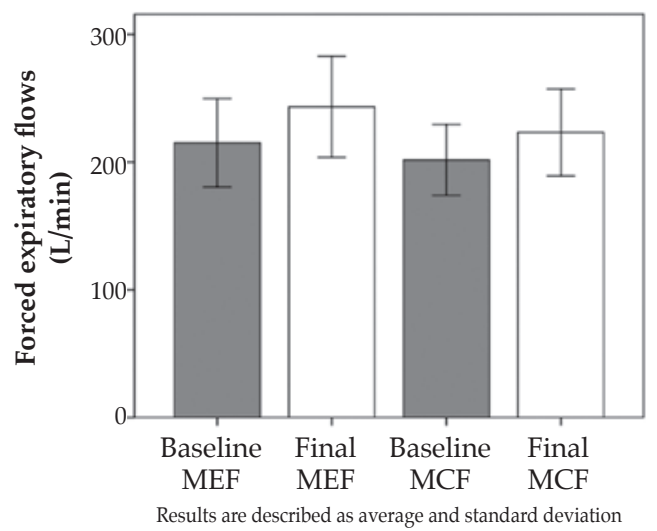

C

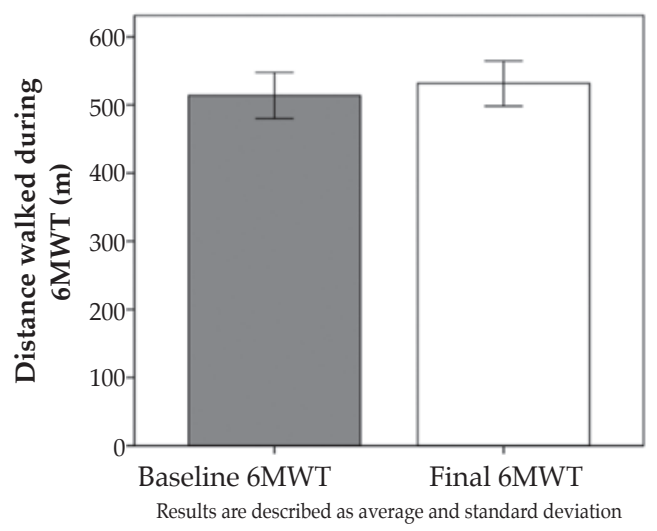

MIP: maximal inspiratory pressure;

MEP: maximal expiratory pressure; MEF: maximal expiratory flow; MCF: maximal cough flow; 6MWT: six-minute walk test.

outcome measures, and 6MWT..$^{12-17}$

The age of the total sample was similar because the program inclusion criteria considered patients 
as of 5 years old with persistent symptoms and limitations; ${ }^{1}$ whereas the severity of disease or specific lung function outcome measures were not considered inclusion criteria for the PR program, which allowed to maintain or improve cardiorespiratory capacity before the progression of impairment. Patient compliance with the program was $65 \%$, except for 3 patients with NMD and 19 with CLD, who lived far away from the facility, had poor compliance with other medical treatments, inexistent regular control of underlying disease before starting the PR, and a socioeconomic situation that limited their access, so their rehabilitation strategy was changed to maintain their compliance.

In our study, MIP increased by $19.3 \%$ and MEP, $14.5 \%$, which was consistent with the findings of Rodríguez et al., who observed improved respiratory muscle strength in children with NMD and CLD after following a home RMT protocol. $^{18}$

In the total group, lung function parameters after the protocol increased by $12.9 \%$ of the absolute FVC and $11.9 \%$ of the absolute $\mathrm{FEV}_{1}$. Prior studies have shown inconsistent results in relation to the impact of PR protocols on these outcome measures. Colom et al., demonstrated that forced expiratory volumes were greater, but FVC increased disproportionately in relation to $\mathrm{FEV}_{1}$ and the $\mathrm{FEV}_{1} / \mathrm{FVC}$ ratio decreased. Therefore, it is not possible to rule out that the observed changes may be related to phenomena associated with children growth or learning ability to perform the tests. ${ }^{19}$

The TDW in the 6MWT increased by $3 \%$ in the total sample, but this was not statistically

Table 3. Spirometry at baseline and 3 months after admission for the total group with training $(n=102)$, described as mean \pm standard deviation

\begin{tabular}{lccc}
\hline Outcome measures & Baseline assessment & Assessment after 3 months & $p$ value \\
\hline FVC $(\mathrm{L})$ & $1.91 \pm 0.95$ & $2.15 \pm 1.23$ & $0.001^{*_{\mathrm{a}}}$ \\
$\mathrm{FVC}(\%)$ & $87.7 \pm 27.7$ & $87.2 \pm 26.2$ & 0.847 \\
$\mathrm{FEV}_{1}(\mathrm{~L})$ & $1.51 \pm 0.70$ & $1.69 \pm 0.94$ & $0.004^{\text {*a }_{a}}$ \\
$\mathrm{FEV}_{1}(\%)$ & $77.9 \pm 25.8$ & $77.1 \pm 25.2$ & $0.664^{\mathrm{a}}$ \\
$\mathrm{FEV}_{1} / \mathrm{FVC}$ & $77.4 \pm 22.2$ & $75.7 \pm 18.5$ & $0.018^{\text {*a }_{a}}$ \\
$\mathrm{FEF}_{25-75}(\mathrm{~L} / \mathrm{min})$ & $1.62 \pm 0.79$ & $1.72 \pm 1.07$ & $0.328^{\mathrm{a}}$ \\
$\mathrm{FEF}_{25-75}(\%)$ & $66.7 \pm 33.5$ & $68.4 \pm 39.1$ & $0.636^{\mathrm{a}}$ \\
\hline
\end{tabular}

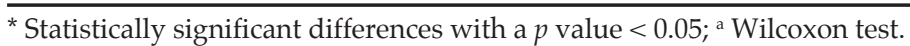

TABLE 4. Assessment at baseline and 3 months after admission for the neuromuscular disease and chronic lung disease groups, described as mean \pm standard deviation

\begin{tabular}{|c|c|c|c|c|c|c|}
\hline \multirow[t]{2}{*}{ Outcome measures } & \multicolumn{3}{|c|}{$\begin{array}{l}\text { NMD } \\
(\mathrm{n}=35)\end{array}$} & \multicolumn{3}{|c|}{$\begin{array}{c}\text { CLD } \\
(\mathrm{n}=49)\end{array}$} \\
\hline & Pre- & Post- & $p$ value & Pre- & Post- & $p$ value \\
\hline$\overline{F V C ~(L) ~}$ & $1.68 \pm 0.69$ & $1.71 \pm 0.74$ & 0.704 & $2.20 \pm 1.06$ & $2.60 \pm 1.40$ & $0.001^{*}$ \\
\hline $\operatorname{FVC}(\%)$ & $84.9 \pm 33.5$ & $79.85 \pm 28.2$ & 0.252 & $92.7 \pm 21.6$ & $93.6 \pm 23.6$ & 0.748 \\
\hline $\mathrm{FEV}_{1}(\mathrm{~L})$ & $1.51 \pm 0.54$ & $1.53 \pm 0.59$ & $0.530^{\mathrm{a}}$ & $1.63 \pm 0.79$ & $1.96 \pm 1.08$ & $0.001^{*_{a}}$ \\
\hline $\operatorname{FEV}_{1}(\%)$ & $83.4 \pm 30.8$ & $78.5 \pm 24.0$ & 0.267 & $76.1 \pm 21.7$ & $78.6 \pm 25.8$ & $0.676^{a}$ \\
\hline $\mathrm{FEV}_{1} / \mathrm{FVC}$ & $89.6 \pm 8.3$ & $88.7 \pm 8.5$ & 0.496 & $75.0 \pm 13.0$ & $71.7 \pm 13.0$ & $0.005^{*}$ \\
\hline $\mathrm{FEF}_{25-75}(\mathrm{~L} / \mathrm{min})$ & $1.97 \pm 0.70$ & $2.03 \pm 0.84$ & 0.606 & $1.49 \pm 0.87$ & $1.59 \pm 1.18$ & $0.566^{\mathrm{a}}$ \\
\hline $\mathrm{FEF}_{25-75}^{25-7 /}(\%)$ & $93.6 \pm 29.2$ & $95.0 \pm 38.7$ & $0.433^{\mathrm{a}}$ & $53.7 \pm 31.9$ & $56.8 \pm 35.6$ & $0.663^{\mathrm{a}}$ \\
\hline $\operatorname{MIP}\left(\mathrm{cmH}_{2} \mathrm{O}\right)$ & $55.3 \pm 19.7$ & $61.6 \pm 20.8$ & 0.093 & $66.2 \pm 19.0$ & $77.8 \pm 21.8$ & $0.006^{*_{a}}$ \\
\hline $\operatorname{MIP}(\%)$ & $51.7 \pm 17.5$ & $58.4 \pm 18.3$ & 0.093 & $64.3 \pm 19.8$ & $76.5 \pm 24.6$ & $0.009^{*}$ \\
\hline $\operatorname{MEP}\left(\mathrm{cmH}_{2} \mathrm{O}\right)$ & $42.6 \pm 18.4$ & $50.29 \pm 19.8$ & $0.014^{*}$ & $70.5 \pm 21.6$ & $75.7 \pm 21.6$ & 0.239 \\
\hline $\operatorname{MEP}(\%)$ & $32.2 \pm 14.4$ & $37.0 \pm 15.4$ & $0.126^{\mathrm{a}}$ & $52.6 \pm 17.0$ & $55.8 \pm 16.5$ & 0.291 \\
\hline $\operatorname{MEF}(\mathrm{L} / \mathrm{min})$ & $150.4 \pm 51.3$ & $178.5 \pm 53.4$ & $0.021^{*}$ & $230.3 \pm 107.2$ & $251.7 \pm 130.9$ & 0.062 \\
\hline $\operatorname{MEFc}(\mathrm{L} / \mathrm{min})$ & $165.0 \pm 56.4$ & $165.0 \pm 62.5$ & 1.000 & $217.7 \pm 84.2$ & $248.5 \pm 108.5$ & 0.070 \\
\hline 6MWT (m) & $359.8 \pm 138.2$ & $376.4 \pm 147.4$ & 0.445 & $564.6 \pm 89.7$ & $582.0 \pm 87.5$ & 0.073 \\
\hline $6 \mathrm{MWT}(\%)$ & $62.3 \pm 18.9$ & $61.1 \pm 22.8$ & 0.742 & $67.0 \pm 20.7$ & $70.948 \pm 20.6$ & 0.150 \\
\hline
\end{tabular}

MIP: maximal inspiratory pressure; MEP: maximal expiratory pressure; MEF: maximal expiratory flow;

MEFc: maximal expiratory flow coughed; 6MWT: six-minute walk test; NMD: neuromuscular disease; CLD: chronic lung disease.

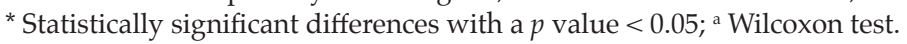


significant in the disease sub-groups. These results were consistent with those of Rodríguez et al., ${ }^{20}$ in NMD patients, but not with those of Latorre-Román et al., ${ }^{21}$ who observed improvements in the TDW in the 6MWT in asthma children.

Specifically, patients with NMD showed, after 10 months of training, that inspiratory and expiratory muscle strength increased by $45.5 \%$ and $37.5 \%$, respectively, ${ }^{18}$ which reached a plateau at 10 months of training. ${ }^{12}$ This is not consistent with our results, which showed an increase in expiratory muscle strength only. A new assessment of respiratory muscle strength after a short 3-month period of RMT may have underestimated the outcomes of the protocol performed in our facility. Such early reassessment was done because, as of 3 months, many patients changed their rehabilitation strategy to a mixed or home protocol, which resulted in more heterogeneous outcomes.

In our study, patients with NMD did not show significant changes in the spirometry parameters or the TDW in the 6MWT, as described in other articles. ${ }^{22-23}$ This was consistent with the results of the study by Rodríguez et al. ${ }^{20}$ in patients with progressive NMD, who did not observe a longer TDW in the 6MWT; however, they corroborated a significant reduction in perceived exertion and leg fatigue after a GPT protocol. It has been suggested that outcome measures like quality of life and oxygen consumption improved significantly after physical training-based interventions, with no evidence of exercise-induced muscle injury. ${ }^{24}$

Studies in patients with CLD have confirmed the positive impact of RMT, with improvements in respiratory muscle strength after a training protocol. ${ }^{13,15,25}$ Rodríguez et al. observed variations of $33.3 \%$ in both the MIP and the MEP of CLD patients after 10 months of RMT. ${ }^{20}$ In our study, patients with CLD showed a $17.9 \%$ improvement in the absolute MIP.

These patients experienced favorable changes in spirometry outcome measures. It has been demonstrated that GPT prevents a decrease in lung function in long-term rehabilitation protocols. ${ }^{26-28}$

Our study has several limitations, for example, the heterogeneous population prevented us from making conclusions on the behavior of a specific disease, which resulted in dispersed outcomes.

The absence of a control group and blinded measurements hindered the possibility of ruling out the potential influence of children's learning abilities to perform the tests, which may have overestimated results. Some of the observed associations were not statistically significant; however, the absolute relevance of such differences was consistent with the results of prior studies. Therefore, we cannot rule out the fact that the sample size of this case series may have not been enough to reduce the risk for type II error in some of the analysis.

Finally, we may conclude that children and adolescents with chronic conditions had a lower cardiorespiratory functional capacity and that those who were admitted to the rehabilitation protocol experienced an increase in their respiratory muscle strength and physical capacity. Future controlled studies are required to confirm such results.

\section{REFERENCES}

1. Torres-Castro R, Zenteno D, Rodríguez-Núñez I, Villarroel $\mathrm{G}$, et al. Guías de rehabilitación respiratoria en niños con enfermedades respiratorias crónicas: Actualización 2016. Neumol Pediatr. 2016; 11(3):114-31.

2. Zenteno D, Puppo H, Vera R, Torres R, et al. Guías de rehabilitación para niños con enfermedades respiratorias crónicas. Neumol Pediatr. 2008; 3(supl):25-33.

3. Spruit MA, Singh SJ, Garvey C, ZuWallack R, et al. An official American Thoracic Society/European Respiratory Society statement: key concepts and advances in pulmonary rehabilitation. Am J Respir Crit Care Med. 2013; 188(8):e13-64.

4. Linares M, Sánchez I, Corrales R, Díaz A, et al. Pruebas de función pulmonar en el niño. Rev Chil Pediatr. 2000; 71(3):228-42.

5. ATS Committee on Proficiency Standards for Clinical Pulmonary Function Laboratories. ATS statement: guidelines for the six-minute walk test. Am J Respir Crit Care Med. 2002; 166(1):111-7.

6. Rodríguez I, Zenteno D, Cisternas L, Rodríguez P, et al. Construcción y evaluación de EPInfant: una escala para la medición del esfuerzo percibido en la población pediátrica. Arch Argent Pediatr. 2015; 113(6):550-7.

7. Gatica D, Puppo H, Villarroel G, San Martín I, et al. Valores de referencia del test de marcha de seis minutos en niños sanos. Rev Med Chile. 2012; 140(8):1014-21.

8. American Thoracic Society/European Respiratory Society. ATS/ERS Statement on respiratory muscle testing. Am J Respir Crit Care Med. 2002; 166(4):518-624.

9. Contreras I, Vidal F, Caussade S, Sánchez I, et al. Valores normales de presión inspiratoria y presión espiratoria máxima en niños y adolescentes chilenos sanos. Neumol Pediatr. 2007; 2(3):163.

10. Miller MR, Hankinson J, Brusasco V, Burgos F, et al. Standardisation of spirometry. Eur Respir J. 2005; 26(2): 319-38.

11. Céspedes GJ, Gutiérrez CM, Oyarzún GM. Flujometría en la práctica de atención primaria. Rev Chil Enferm Respir. 2010; 26(1):47-8.

12. Koessler W, Wanke T, Winkler G, Nader A, et al. 2 years' experience with inspiratory muscle training in patients with neuromuscular disorders. Chest. 2001; 120(3):765-9.

13. Houston BW, Mills N, Solis-Moya A. Inspiratory muscle training for cystic fibrosis. Cochrane Database Syst Rev. 2013; (11):CD006112.

14. De Jong W, Van Aalderen W, Kraan J, Koeter G, et al. 
Inspiratory muscle training in patients with cystic fibrosis. Respir Med. 2001; 95(1):31-6.

15. Silva J, PuppoH, Vargas D, González R, et al. Entrenamiento muscular inspiratorio en niños secuelados por adenovirus. Rev Chil Enferm Respir. 1999; 15:239.

16. Gozal D, Thiriet P. Respiratory muscle training in neuromuscular disease: long-term effects on strength and load perception. Med Sci Sports Exerc. 1999; 31(11):1522-7.

17. Topin N, MateckiS, LeBris S, RivierF, et al. Dose-dependent effect of individualized respiratory muscle training in children with Duchenne muscular dystrophy. Neuromuscul Disord. 2002; 12(6):576-83.

18. Rodríguez Núñez I, Zenteno Araos D, Manterola Delgado C. Effects of home-based respiratory muscle training in children and adolescent with chronic lung disease. J Bras Pneumol. 2014; 40(6):626-33.

19. Colom AJ, Maffey A, Garcia Bournissen F, Teper A. Pulmonary function of a paediatric cohort of patients with postinfectious bronchiolitis obliterans. A long term followup. Thorax. 2015; 70(2):169-74.

20. Rodríguez Núñez I, Fuentes SC, Rivas BC, Molina RF, et al. Rehabilitación respiratoria en el paciente neuromuscular: efectos sobre la tolerancia al ejercicio y musculatura respiratoria. Resultado de una serie de casos. Rev Chil Enferm Respir. 2013; 29(4):196-203.

21. Latorre-Román P, Navarro-Martínez V, García-Pinillos F. The effectiveness of an indoor intermittent training program forimproving lung function, physical capacity, body composition and quality of life in children with asthma. $J$ Asthma. 2014; 51(5):544-51.

22. Khirani S, Colella M, Caldarelli V, Aubertin G, et al. Longitudinal course of lung function and respiratory muscle strength in spinal muscular atrophy type 2 and 3. Eur J Paediatr Neurol. 2013; 17(6):552-60.

23. KhiraniS, Ramirez A, Aubertin G, BouléM, etal. Respiratory muscle decline in Duchenne muscular dystrophy. Pediatr Pulmonol. 2014; 49(5):473-81.

24. Aboussouan LS. Mechanisms of exercise limitation and pulmonary rehabilitation for patients with neuromuscular disease. Chron Respir Dis. 2009; 6(4):231-49.

25. Taivassalo T, De Stefano N, Chen J, Karpati G, et al. Short term aerobic training response in chronic myopathies. Muscle Nerve. 1999; 22(9):1239-43.

26. Lötters F, Van Tol B, Kwakkel G, Gosselink R. Effects of controlled inspiratory muscle training in patients with COPD: a meta-analysis. Eur Respir J. 2002; 20(3):570-6.

27. Van Doorn N. Exercise programs for children with cystic fibrosis: a systematic review of randomized controlled trials. Disabil Rehabil. 2010; 32(1):41-9.

28. Eichenberger PA, Diener SN, Kofmehl R, Spengler CM. Effects of exercise training on airway hyperreactivity in asthma: a systematic review and meta-analysis. Sports Med. 2013; 43(11):1157-70. 